\title{
Energetics and electronic structure of graphene nanoribbons under a lateral electric field
}

\author{
Ayaka Yamanaka*, Susumu Okada** \\ Graduate School of Pure and Applied Sciences, University of Tsukuba, 1-1-1 Tennodai, Tsukuba, \\ Ibaraki 305-8571, Japan
}

\begin{abstract}
We studied the energetics and electronic structure of graphene nanoribbons with hydrogenated and clean edges with respect to the detailed edge shapes using density functional theory. Our calculations showed that the stability of graphene edges strongly depends on the length of the zigzag edge portion. Near-zigzag edges are less stable than near-armchair edges because of the large number of states at the Fermi level $\left(E_{F}\right)$ in nanoribbons with near-zigzag edges. The edge formation energy retains a constant value up to the edge angle of $16^{\circ}$, after which it monotonically increases with increasing zigzag portion or edge angle. We also found that the edge stability strongly correlates with the electronic structures near the $E_{F}$ of graphene nanoribbons. Nanoribbons with a small zigzag portion already possess edge localized $\pi$ electronic states near the $E_{F}$. We also demonstrate that a lateral electric field increases the energy gap of ribbons with/without hydrogen termination, resulting in a decrease in edge formation energy.
\end{abstract}

Keywords:

\footnotetext{
*TEL/FAX: +81-298535600 (ext. 8233)/+81-298535924

**TEL/FAX: +81-298535921/+81-298535924

Email addresses: ayamanaka@comas.frsc.tsukuba.ac.jp (Ayaka Yamanaka), sokada@comas.frsc.tsukuba.ac.jp (Susumu Okada) 
PACS: 61.48.Gh, 68.35.-p, 73.22.Pr

\section{Introduction}

In recent years, graphene has attracted much attention in the fields of nanoscience and nanotechnology because of its unique electronic and geometric properties [1, 2]. A honeycomb network of $\mathrm{sp}^{2} \mathrm{C}$ atoms with single-atom thickness causes a pair of linear dispersion bands at the Fermi level $\left(E_{F}\right)$ [3], leading to peculiar physical properties such as the unusual quantum Hall effect $[4,5,6,7]$. On the other hand, the electronic structure of graphene is known to be fragile with respect to foreign materials such as insulating substrates $[8,9,10]$, metal electrodes $[11,12]$, other graphene/graphite layers $[13,14,15,16]$, and an external electric field $[17,18,19,20,21,22]$. For example, graphene has an energy gap of a few tens of meV when adsorbed on $\mathrm{SiO}_{2}$ surfaces [9]. Bilayer graphene possesses quadric dispersion bands at the $E_{F}$ with zero band gap, rather than the linear dispersion band, that are tunable from metallic to semiconducting by applying a normal electric field $[17,18,19,20,21,22]$. In addition to the hybrid structure perpendicular to the layer, hybrid structures within the layer also affect their electronic properties. Atomic defects and edges are regarded as negative foreign atoms, which strongly modulate the electronic structure of graphene $[23,24,25,26,27,28]$. Furthermore, heterogeneous sheets with h-BN also modulate the characteristic electronic structure of graphene [29]. These are serious problems when incorporating graphene as an emerging material in future nanoscale devices because these hybrid structures are inherent and essential in such devices.

To utilize graphene for the devices, it is important to precisely control the ge- 
ometric and electronic structures of graphene under hybrid structures with foreign materials and external environments. The geometric and electronic structures of graphene edges have been intensively studied for the past two decades because edges are the one of important procedures for electronic structure tuning and functionalization of graphene [30]. It is well known that graphene nanoribbons possess peculiar electronic structures depending on their width and edge atomic arrangements: graphene nanoribbons with zigzag edges possess edge localized states known as edge states [23, 24, 25, 26, 29] arising from the topological condition, while ribbons with armchair edges have either metallic or semiconducting properties depending on the discretized conditions imposed on graphene. Additionally, the edge roughness induces further variations in their electronic structures $[31,32]$. In addition to their electronic structures, the energetics of graphene nanoribbons in terms of the edge structures has been theoretically studied. Previous theoretical works have pointed out that the armchair edge is energetically more stable than the zigzag edge because of the small density of states at the $E_{F}[32,33,34,35,36,37]$. However, despite much effort devoted to elucidating the energetics and electronic structure of the edges of graphene [32, 33, 34, 35, 36, $37,38]$, the correlation between energetics and electronic structures of graphene nanoribbons with respect to the edge atomic structures is still not fully understood.

In this paper, we aim to elucidate the correlation between the edge shape and physical properties of graphene nanoribbons using first-principles total energy calculations based on density functional theory. For this purpose, we study the edge stability and the electronic structures of graphene nanoribbons with various edge shapes, from armchair to zigzag edges, with and without hydrogen termination. In addition, we also investigate the energetics and electronic structure of 
graphene nanoribbons with various edge angles under a parallel electric field to give theoretical insight into the fundamental issues for utilizing graphene in electronic devices, because the external electric field is essential for functionalizing graphene nanoribbons as in electronic device structures. Our calculations showed that the energetics and electronic structure of graphene nanoribbons strongly depend on the detailed edge structures. Edge formation energy retains a constant value around armchair edges, while it monotonically increases with increasing a portion of zigzag edges. We also demonstrate that the energetics strongly correlate with the electronic structures of graphene nanoribbons: ribbons with neararmchair edges are semiconductors with a finite energy gap, while ribbons with near-zigzag edges are metals in which the density of states at the $E_{F}$ monotonically increases with increasing a portion of zigzag edges. A parallel electric field decreases the edge formation energy of all graphene nanoribbons studied here. For ribbons with clean edges, because dangling bond states appeared around the $E_{F}$, the edge formation energy monotonically increases with increasing edge angle from armchair $\left(\theta=0^{\circ}\right)$ to zigzag $\left(\theta=30^{\circ}\right)$.

\section{Methods and models}

In this work, we studied the geometric and electronic structures of graphene nanoribbons with various edge shapes using density functional theory [39, 40] implemented into the Simulation Tool for Atom Technology (STATE) [41]. We used the local density approximation with the Perdew-Zunger functional form fitted to the Quantum Monte Carlo results for a homogeneous electron gas to calculate the exchange correlation potentials among interacting electrons [42, 43]. Ultrasoft pseudopotentials generated with the Vanderbilt scheme were used to de- 
scribe electron-ion interactions [44]. Valence wave functions and charge densities were expanded in terms of the plane wave basis set with cutoff energies of 25 and 225 Ry, respectively.

To investigate the electronic structures and energetics of nanoribbons under a lateral electric field, we used the effective screening medium method to solve the Poisson equation including the external electric field [45]. We applied a parallel electric field between two planar metal electrodes simulated by the effective screening medium that were situated alongside the rightmost and leftmost atoms of the nanoribbons with $8 \AA$ vacuum spacings.

In this work, we considered several edge structures between armchair and zigzag to investigate their energetics and electronic structures without and with external electric field. To simulate graphene edges with various edge shapes, we constructed nanoribbons with hydrogenated and clean edges with edge angles of 0 (armchair), 8, 16, 23, and $30^{\circ}$ (zigzag) . To exclude the width dependence of the energetics, all ribbons possess almost the same width, of approximately $20 \AA$. The atomic structures were fully optimized under a zero electric field until the force acting on each atom was less than $5 \mathrm{mRy} / \AA$. During calculations under the condition of a finite electric field of $0.25 \mathrm{~V} / \AA$, the geometries were fixed to those under the condition of a zero electric field.

\section{Results and discussion}

\subsection{Hydrogenated edges}

Figure 1 shows the optimized structures of hydrogenated graphene nanoribbons with various edge angles. In all cases, the bond lengths of nanoribbons are not equivalent to each other: Covalent bonds associated with hydrogenated $\mathrm{C}$ 
(a) $\theta=0^{\circ}$

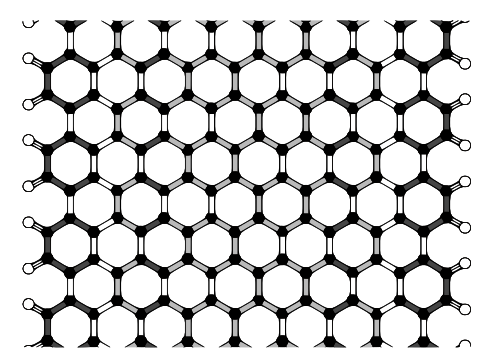

(c) $\theta=16^{\circ}$

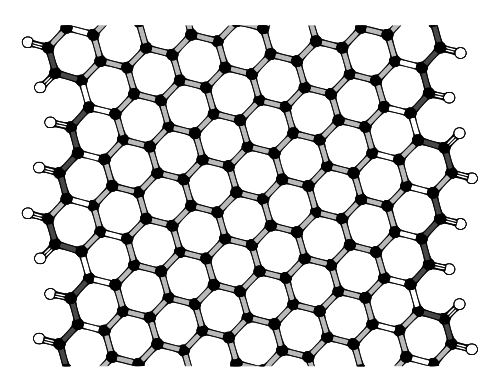

(e) $\theta=30^{\circ}$

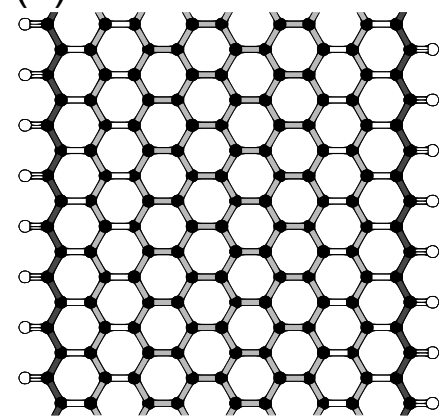

(b) $\theta=8^{\circ}$

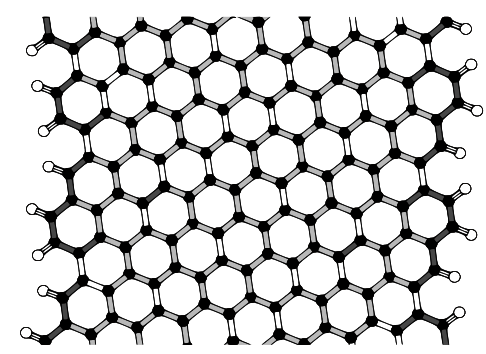

(d) $\theta=23^{\circ}$

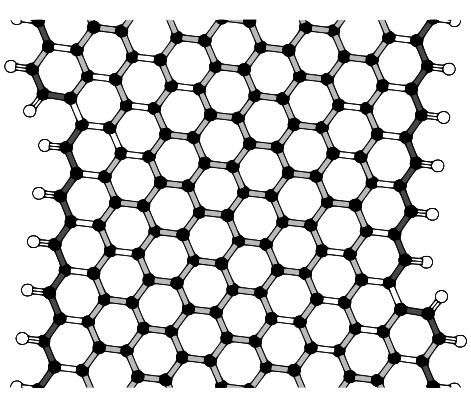

Figure 1: Optimized geometries of graphene nanoribbons with (a) armchair edge $\left(\theta=0^{\circ}\right)$, chiral edges (b) $\theta=8^{\circ}$, (c) $\theta=16^{\circ}$, (d) $\theta=23^{\circ}$, and (e) zigzag edge $\left(\theta=30^{\circ}\right)$. Black and white circles denote carbon and hydrogen atoms, respectively. Dark shaded, pale shaded, and white bonds indicate short (-1.41 $\mathrm{A})$, medium (1.41-1.42 $\mathrm{A})$, and long (1.42 $\mathrm{A}-$ ) bonds, respectively. Stripe bonds situated at the edge of the nanoribbons correspond to $\mathrm{C}-\mathrm{H}$ bonds. 
atoms are found to be shorter than the other bonds. The bond lengths are $1.37 \AA$ or shorter. This short bond length is ascribed to the environment arising from the hydrogen atoms attached to the $\mathrm{C}$ atoms. In this case, because of the chemical species difference, one of three bonds of the $\mathrm{C}$ atoms is not equivalent to the remaining two. The bonds adjacent to these short bonds are basically longer than the initial bond length of $1.42 \AA$. For ribbons with near-armchair edges, there is a clear bond alternation with respect to the $\mathrm{C}_{2}$ dimer rows along the ribbon direction. This bond alternation leads to stepwise screening against the external electric field, which will be discussed later. In contrast, for ribbons with near-zigzag edges, bond alternation rapidly decreases approaching the inner region of the ribbons. Indeed, the bond length at the center of the ribbons is almost equivalent to the initial length of $1.42 \AA$.

Figure 2 (a) shows the edge formation energy of hydrogenated graphene nanoribbons as a function of the edge angle. The edge formation energy $E_{\text {edge }}$ is evaluated using the following formula:

$$
E_{\text {edge }}=\left(E_{\text {total }}-N_{\mathrm{C}} \mu_{\mathrm{C}}-N_{\mathrm{H}} \mu_{\mathrm{H}}\right) / L_{\text {edge }}
$$

where $E_{\text {total }}, N_{\mathrm{C}}, N_{\mathrm{H}}, \mu_{\mathrm{C}}, \mu_{\mathrm{H}}$, and $L_{\text {edge }}$ denote the total energy of ribbons, the number of $\mathrm{C}$ atoms, the number of $\mathrm{H}$ atoms, the chemical potential of $\mathrm{C}$ atoms, the chemical potential of $\mathrm{H}$ atoms, and the edge length of a unit cell, respectively. The chemical potentials of $\mathrm{C}$ and $\mathrm{H}$ atoms are evaluated by the total energies per atom of graphene and $\mathrm{H}$ in benzene molecules, respectively. As shown in Fig. 2 (a), the edge formation energy remains constant up to $\theta=16^{\circ}$. Because the edge formation energy remains constant up to the edge angle of $16^{\circ}$, the graphene flakes possess armchair edges with a small portion of zigzag edges. This result implies that the armchair edges of graphene inherently possess edge roughness, contain- 


\section{(a) Formation Energy}

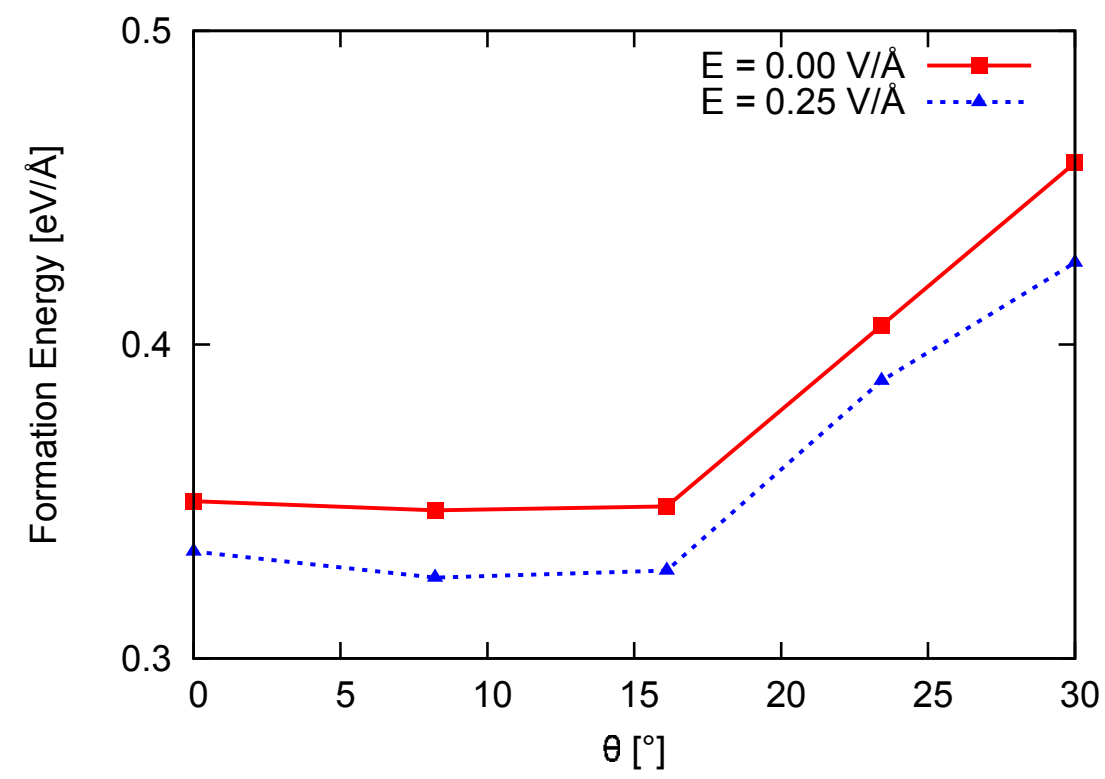

(b) Energy Gap

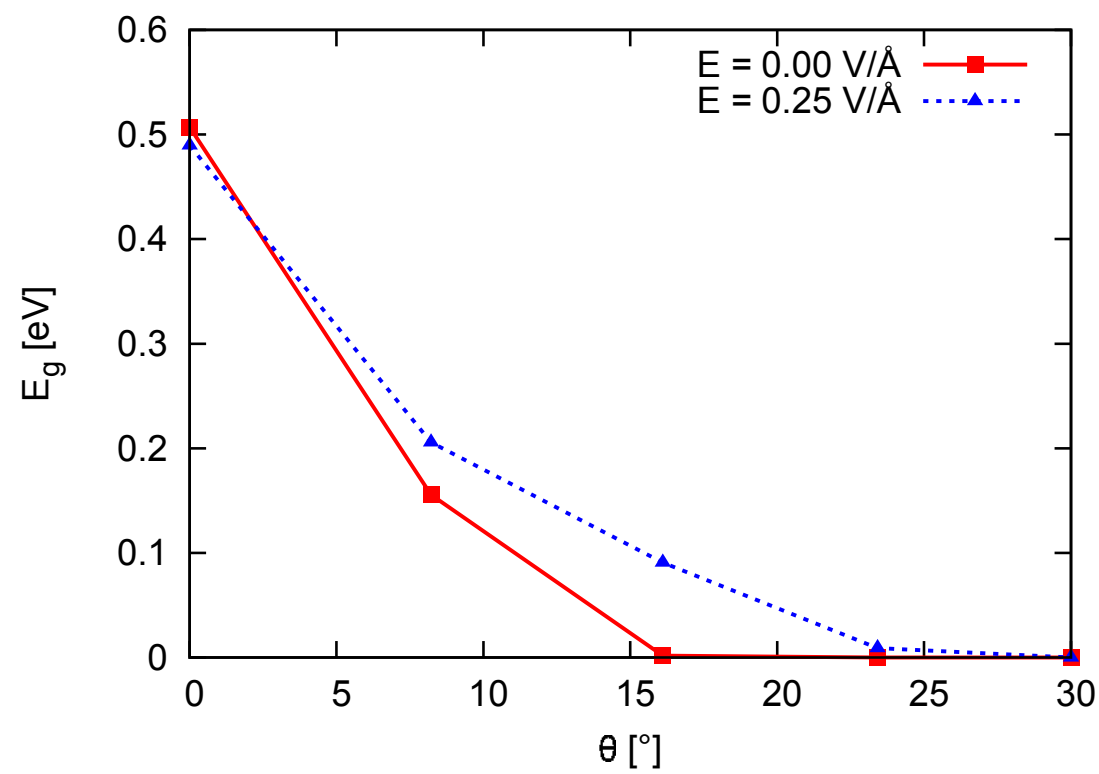

Figure 2: (Color online) (a) Edge formation energy and (b) energy gap of hydrogenated graphene nanoribbons as a function of the edge angle $\theta$. Solid and dotted lines denote the edge formation energy and energy gap without and with electric field, respectively. 
ing zigzag edges under thermal equilibrium conditions. In sharp contrast, above the critical angle of $\theta=16^{\circ}$, the edge formation energy rapidly increases with increasing edge angle, so that the edge formation energy sensitively correlates with the portion of perfect zigzag edges [38]. This fact corroborates that the edges of graphene prefer armchair or near-armchair shapes over a zigzag shape.

It is well known that the graphene nanoribbons with zigzag edges exhibit spin polarization at the edge atomic sites because of the edge states. To check the spin polarization effects on the edge formation energy of ribbons, we study the energetics of graphene nanoribbons by using the local spin density approximation. Our calculations show that the ribbons with their edge angles of $\theta=16^{\circ}$ or larger possess spin polarization near the edge atomic sites. According to this polarization, edge formation energy calculated by the local spin density approximation slightly decreases of $10 \mathrm{meV}$ or less compared with that obtained by the local density approximation. Therefore, spin degree of freedom weakly affect the energetics of graphene nanoribbons with hydrogenated edges.

When we applied the electric field, the edge formation energy was slightly modulated for all cases. Near the armchair edges, the formation energies at the edge angle of $\theta=8$ and $16^{\circ}$ are smaller than that of the perfect armchair edge. This fact indicates that the lateral electric field enhances the edge roughness of graphene edges. More interestingly, the energy of the zigzag edge substantially decreases with applying electric field. Therefore, the parallel electric field increases the relative stability of the zigzag edges of graphene flakes and nanoribbons.

It is worth to mention the effect of electric field on the polarized electron spin on graphene nanoribbons. Our calculations show that the ribbons with the edge 
angles of $23^{\circ}$ and $30^{\circ}$ still exhibit the spin polarization. On the other hand, the ribbon with edge angle of $16^{\circ}$ does not exhibit spin polarization because of its semiconducting electronic structure under the electric field. Thus, the facts indicate that the polarized electron spin associated with the edge states is robust against the lateral electric field applied in this work.

Figure 2 (b) shows the energy gap between the highest occupied (HO) and the lowest unoccupied (LU) states of graphene nanoribbons studied here. We found that graphene nanoribbons with edge angles up to $15^{\circ}$ are semiconductors. From armchair to the edge angle of $15^{\circ}$, the band gap monotonically decreases with increasing edge angle. Finally, at $16^{\circ}$, the graphene nanoribbon is a zero gap semiconductor. In contrast, nanoribbons with near-zigzag edges are metals. By comparing Figs. 2 (a) and 2 (b), we found a correlation between the edge formation energy and the energy gap: semiconducting graphene nanoribbons have small edge formation energy. It should be noted that the edge formation energy is insensitive to the detailed electronic structure of the graphene nanoribbons with a finite energy gap [34]. On the other hand, metallic graphene nanoribbons have higher edge formation energy. Thus, the energetics of graphene edges depends on their detailed electronic structures.

By applying the electric field, the energy gap basically increases compared with that of the graphene nanoribbons without an electric field, except for the armchair and zigzag edges. It has been reported that a lateral electric field can modulate the electronic structure of graphene nanoribbons with zigzag edges [46, 47]. As for ribbons with edge angles of 8 and $16^{\circ}$, the energy gap under the electric field is wider than that without the field. This gap enlargement with the electric field may offer a practical route to enhance the relative stability of these edges. 

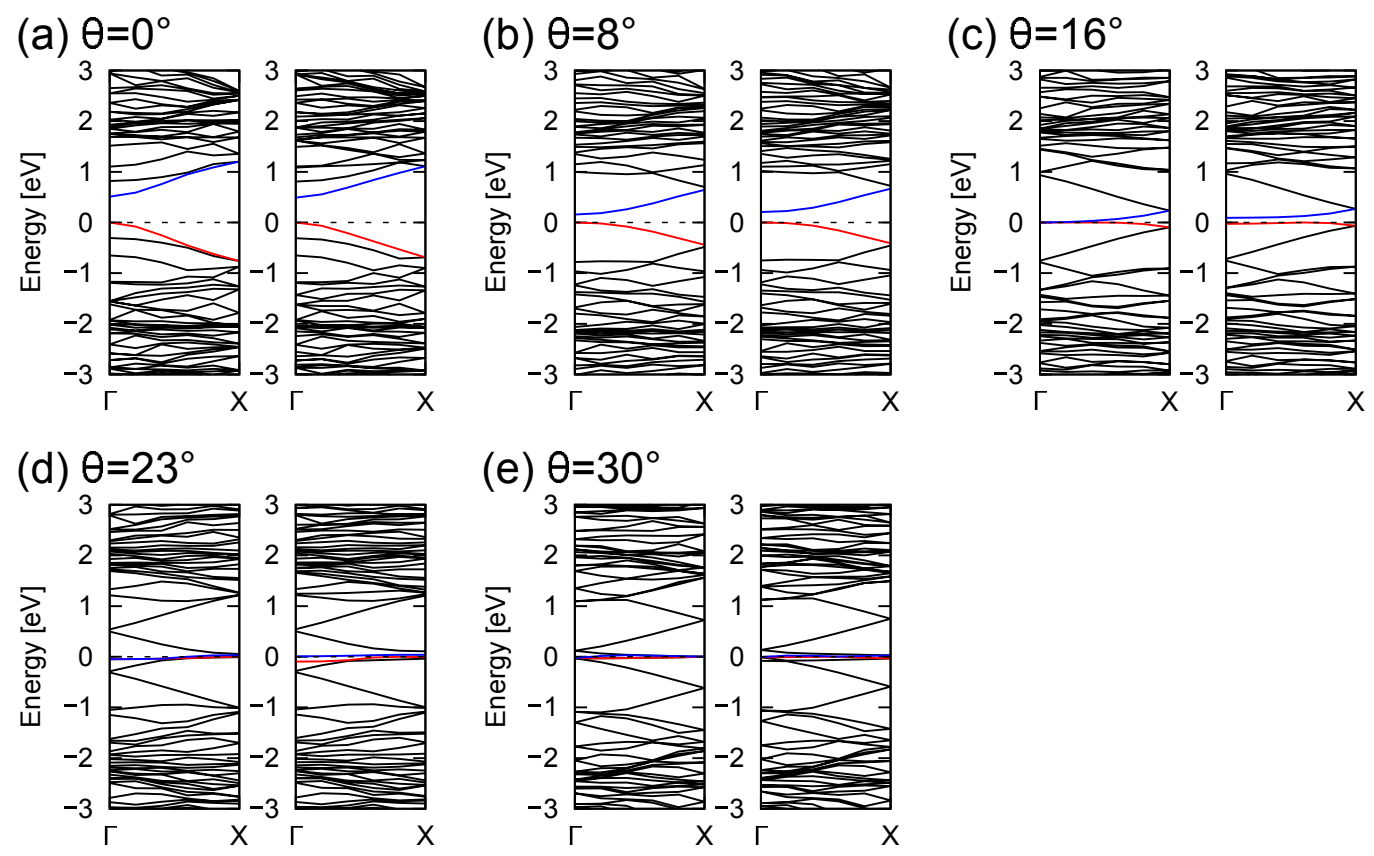

Figure 3: (Color online) Electronic structure of hydrogenated graphene nanoribbons without (left panel) and with (right panel) an electric field. Red and blue lines indicate the highest occupied state and lowest unoccupied state, respectively. Energies are measured from the $E_{F}$ and the top of the valence bands for metallic and semiconducting nanoribbons, respectively.

Although the applied electric field is large, the gap modulation is in the order of $0.1 \mathrm{eV}$. This small modulation is ascribed to the screening effect of $\pi$ electrons in graphene nanoribbons, leading to the nearly flat electrostatic potential profile inside the ribbons [48]. For the remaining ribbons, gap modulation stabilized the edge formation of graphene.

Figure 3 shows the electronic structure of hydrogenated graphene nanoribbons with various edge angles. The HO and LU states lose their dispersion with increasing edge angle. We found a pair of flat bands around the $\Gamma$ point at the $E_{F}$ for graphene nanoribbons of which edge angle $\theta$ equals to or larger than $16^{\circ}$. 
The flat band region monotonically increases with increasing edge angle. Indeed, in the ribbon with the edge angle of $\theta=23^{\circ}$, the $\mathrm{HO}$ and LU states are perfectly degenerated and have flat dispersion throughout the Brillouin zone. In the case of $\theta=30^{\circ}$, we found that four flat dispersion bands including $\mathrm{HO}$ and LU states emerge at the $E_{F}$. Thus, the number of states at the $E_{F}$ monotonically increases with increasing edge angle $\theta$ from 16 to $30^{\circ}$. This is the origin of the increase in the edge formation energy of the near-zigzag edge, as the large number of states at the $E_{F}$ leads to instability for the electron system. Note that we impose eight times periodicities along the ribbon direction for the ribbon with zigzag edges to guarantee numerical accuracy for comparing the total energies of ribbons in these calculations. Thus, flat band states associated with the edge states are folded multiple times into the $\Gamma$ point.

The HO and LU states of the armchair ribbon retain almost their initial eigenvalues under the electric field. In contrast, for ribbons with finite edge angles, the HO and LU states shift downward and upward, respectively, by applying the lateral electric field, leading to an increase in the band gap. Because the lateral electric field leads to a difference in the electrostatic potential between two edges of graphene nanoribbons, the shift is ascribed to the distribution of localized states near the edge atomic sites, in contrast to the usual $\pi$ states that extend throughout the nanoribbons.

The flat band nature of the $\mathrm{HO}$ and LU states implies that these states possess an edge state nature $[23,24,25,29]$. To unravel the origin of the flat band states, we investigate the wave function of the electron states near the $E_{F}$. Figure 4 shows the contour plots of squared wave functions of the electronic states at the $\Gamma$ point within the energy range from $E_{F}-\Delta$ to $E_{F}+\Delta$, where $\Delta=0.2$ 
(a) $\theta=0^{\circ}$

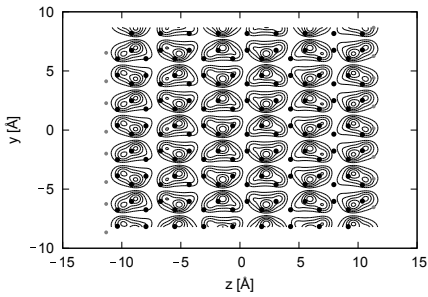

(d) $\theta=23^{\circ}$

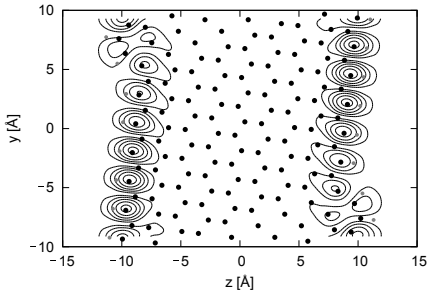

(b) $\theta=8^{\circ}$

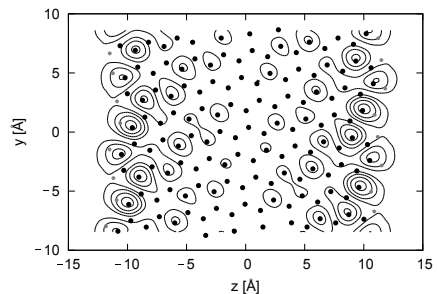

(e) $\theta=30^{\circ}$

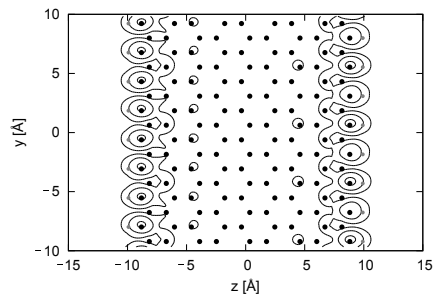

(c) $\theta=16^{\circ}$

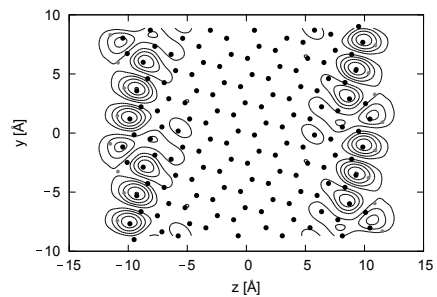

Figure 4: Contour plots of wave functions at the $\Gamma$ point near the $E_{F}$ of hydrogenated graphene nanoribbons.

$\mathrm{eV}$. For graphene nanoribbons with armchair edges, the states extend throughout the ribbon, showing the conventional $\pi$ electron nature. However, the wave functions of the nanoribbons with edge angle $\theta=8^{\circ}$ show a localized nature near the edge atomic site. With further increases in the edge angle, the wave functions are more concentrated at the edge atomic site in the nanoribbons, as in the case of edge angles of $\theta=16$ and $23^{\circ}$, indicating their edge state nature. For graphene nanoribbons with zigzag edges, $\theta=30^{\circ}$, the states are perfectly localized at the edge atomic sites. Therefore, the flat dispersion bands in the graphene nanoribbons with finite edge angles $\theta$ are classified as edge states. These facts indicate that the edge states are basically observed on the various graphene flakes of ribbons in scanning tunneling microscope experiments because the edge roughness is inherent in graphene edges with respect to the energetics. 


\subsection{Clean edges}

We discuss the geometric and electronic structures of graphene nanoribbons with clean edges. Fig. 5 shows the optimized geometries of graphene nanoribbons with clean edges of which angles are $\theta=0$ (armchair), 8, 16, 23, and $30^{\circ}$ (zigzag). In all nanoribbons with any edge angles, structural reconstruction occurs at the edge atoms to reduce electron energy arising from the dangling bonds with increasing lattice energies. In particular, the bonds forming the armchair edge are remarkably shrunken by the structural reconstruction. The calculated bond length is about $1.23 \AA$, possessing an sp nature rather than $\mathrm{sp}^{2}$. This value is consistent with an early calculation on armchair ribbons with clean edges [49]. In the case of zigzag edges, the relaxed bond length near the edges is still shorter than that of conventional $\mathrm{sp}^{2}$ bonds.

Because of the dangling bonds at the edge atomic sites, it is thought that the clean edges are more unstable than the hydrogenated edges. Figure 6 (a) shows the edge formation energies of graphene nanoribbons with clean edges as a function of the edge angles evaluated by the following equation.

$$
E_{\text {edge }}=\left(E_{\text {total }}-N_{\mathrm{C}} \mu_{\mathrm{C}}\right) / L_{\text {edge }}
$$

where $E_{\text {total }}, N_{\mathrm{C}}, \mu_{\mathrm{C}}$, and $L_{\text {edge }}$ denote the total energy of ribbons, the number of C atoms, the chemical potential of $\mathrm{C}$ atoms evaluated with respect to the graphene energy, and the edge length of a unit cell, respectively. As shown in Fig. 6 (a), the formation energy of clean edges is 7 to 8 times larger than that of hydrogenated edges. In contrast to nanoribbons with hydrogenated edges, the edge formation energy monotonically increases with increasing edge angle without any plateaus. It should be noted that the edge formation energy for the ribbons with their edge 
(a) $\theta=0^{\circ}$

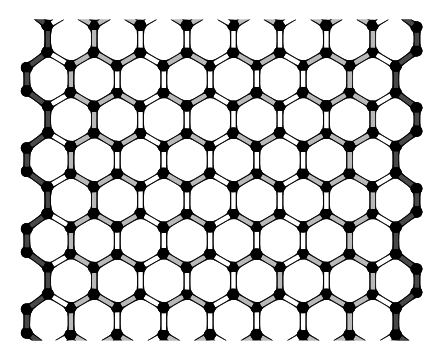

(c) $\theta=16^{\circ}$

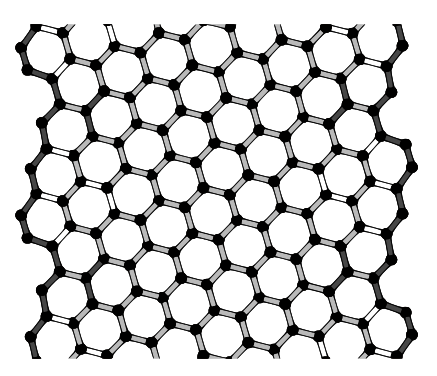

(b) $\theta=8^{\circ}$

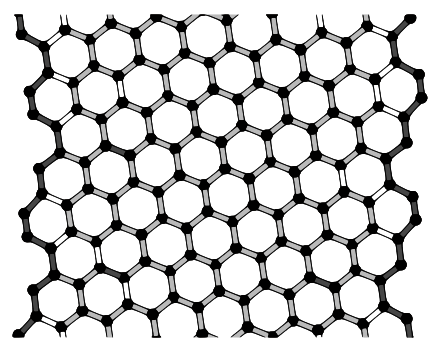

(d) $\theta=23^{\circ}$

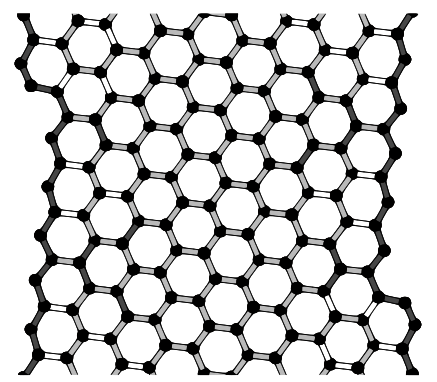

(e) $\theta=30^{\circ}$

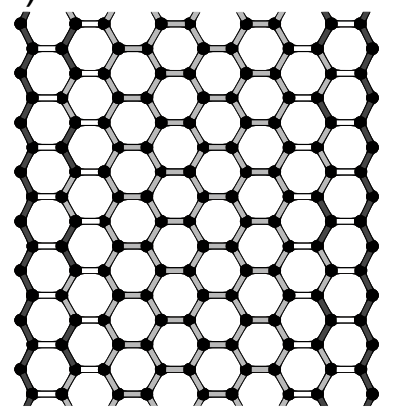

Figure 5: Optimized geometries of graphene nanoribbons with (a) armchair edge $\left(\theta=0^{\circ}\right)$, chiral edges (b) $\theta=8^{\circ}$, (c) $\theta=16^{\circ}$, (d) $\theta=23^{\circ}$, and (e) zigzag edge $\left(\theta=30^{\circ}\right)$. Black and white circles denote carbon and hydrogen atoms, respectively. Dark shaded, pale shaded, and white bonds indicate short ( $-1.41 \AA$ ), medium (1.41-1.42 $)$, and long (1.42 $⿱-$-) bonds, respectively. Striped bonds situated at the edge of the nanoribbons correspond to $\mathrm{C}-\mathrm{H}$ bonds. 


\section{(a) Formation Energy}

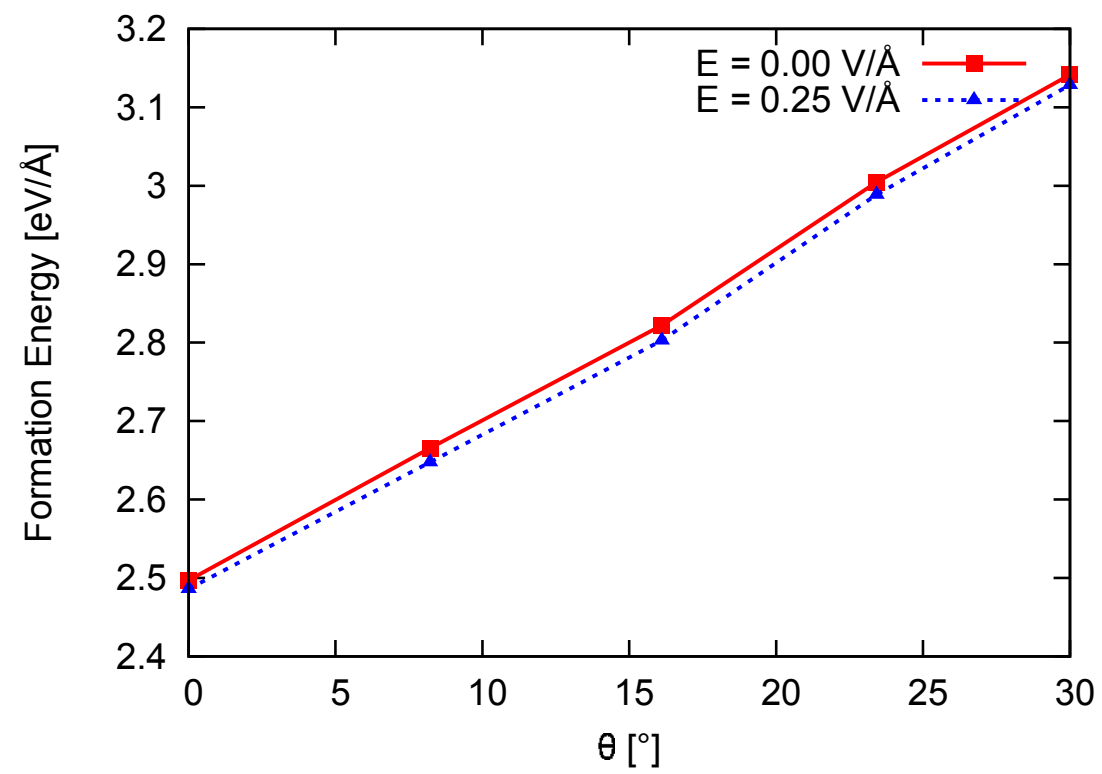

(b) Energy Gap

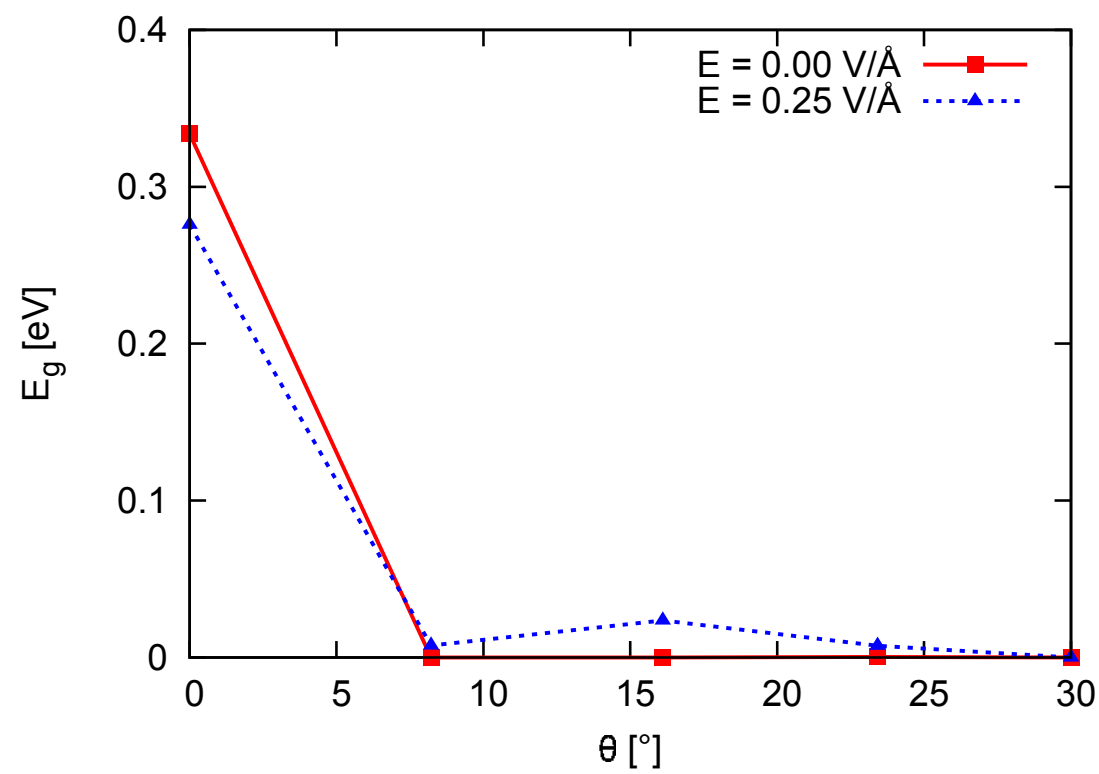

Figure 6: (Color online) (a) Edge formation energy and (b) energy gap of graphene nanoribbons with clean edges as a function of the edge angle $\theta$. Solid and dotted lines denote the edge formation energy and energy gap without and with electric field, respectively. 
angles of $8^{\circ}$ or larger slightly decreases by taking account into the spin degree of freedom, because the spin polarization occurs at edge atomic sites with zigzag shapes caused by the edge states resulting in the decrease of the Fermi level density of states. Edge formation energies obtained by local spin density approximation are smaller than that calculated by the local density approximation by 0.1 $\mathrm{eV} / \AA$. By analogy with the relation between edge formation energies and electronic structure in hydrogenated edges, the monotonic increase of the edge energy implies that nanoribbons with clean edges are metals with a number of electron states near the $E_{F}$.

When we applied the electric field, the edge formation energy slightly decreases irrespective of the edge angles. Modulation of the edge formation energy is almost independent of the edge angles, in sharp contrast to the edge formation energy in hydrogenated nanoribbons.

Figure 6 (b) shows the energy gap of graphene nanoribbons with clean edges. In contrast to nanoribbons with hydrogenated edges, we found that the ribbon with armchair edges is a semiconductor, while the others are all metals. The semiconducting nature of the armchair ribbons makes the armchair edge the most energetically stable edge among the five edge angles. In the case of nanoribbons with armchair edges, as stated above, the edge $\mathrm{C}$ atoms are strongly dimerized with each other, forming a short bond of which length is $1.23 \AA$. Because of this strong dimerization, these atoms possess an sp nature, rather than $\mathrm{sp}^{2}$, so they do not possess dangling bonds even though they are not terminated by $\mathrm{H}$ atoms. By focusing on the detailed edge atomic arrangement for the finite edge angles, we find that the armchair regions of these edges tend to form a C-C dimer with a short bond length of $1.2 \AA$. Thus, the monotonic increase in the edge formation 

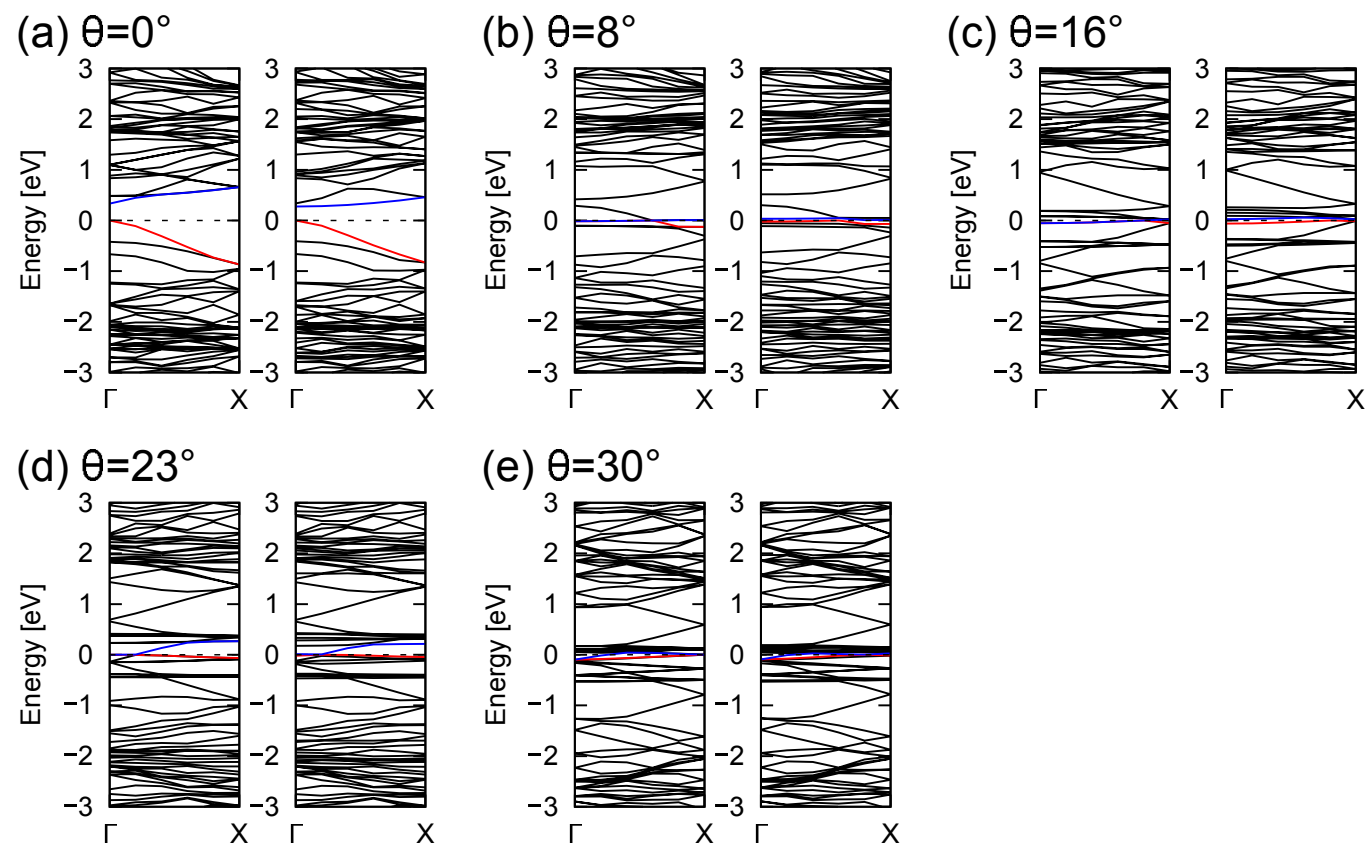

Figure 7: (Color online) Electronic structures of graphene nanoribbons with clean edges without (left panel) and with (right panel) an electric field. Red and blue lines indicate the highest occupied state and lowest unoccupied state, respectively. Energies are measured from the $E_{F}$.

energy is ascribed to the decrease in the amount of $\mathrm{C}-\mathrm{C}$ dimer structures at the edges. Under the lateral electric field, the energy gap slightly increases, except for armchair and zigzag edges. However, the modulation is smaller than that for the hydrogenated edges.

By comparing Figs. 6 (a) and 6 (b), as in the case of hydrogenated edges, we also found a correlation between edge formation energy and energy gap in the case of clean edges. Semiconducting armchair nanoribbons have the smallest edge formation energy. For the other metallic nanoribbons, the edge formation energy is larger than that of the armchair nanoribbon.

Figure 7 shows the electronic structure of nanoribbons with clean edges. Com- 
paring with the electronic structures of nanoribbons with hydrogenated edges, nanoribbons with clean edges have extra states near the $E_{F}$, of which the dispersion is very small, arising from the dangling bonds of $\mathrm{C}$ atoms situated at the edges. Because of the localized nature of the dangling bonds, these states are basically degenerate with respect to each other. As stated above, the dangling bonds are absent at the atomic sites associated with the armchair edges. Thus, the increase in the states corresponds to that of the portion of zigzag edges. Because of the edge states and dangling bond states, the number of states at the $E_{F}$ monotonically increases with increasing edge angle $\theta$ from 8 to $30^{\circ}$. As in the case of hydrogenated nanoribbons, this large number of states at the $E_{F}$ induces instability in edges with angles $\theta$ from 8 to $30^{\circ}$.

Under the lateral electric field, the electronic structure modulation is absent for nanoribbons with clean edges, in sharp contrast to the case of nanoribbons with hydrogenated edges. With clean edges, the number of states leads to a large $E_{F}$ density of states on the nanoribbons, so that the states substantially screen the external electric field and prevent the shift of the electrostatic potential at the edge atomic sites.

For the ribbons with clean edges, large density of states at the $E_{F}$ leads to the spin polarization on the ribbons. Indeed, calculations using the local spin density approximation show the spin density around the edge atomic sites: Distribution of polarized electron spin is the same as that of the dangling bond states, in sharp contrast to the spin density on the ribbons with hydrogenated edges. Accoding to the spin polarization, several dangling bond states split into $\alpha$ and $\beta$ spin bands, leading to the sattelite peaks in a large peak of density of states at the $E_{F}$. On the other hand, remainig states associated with usual $\pi$ states retain their spec- 
(a) $\theta=0^{\circ}$

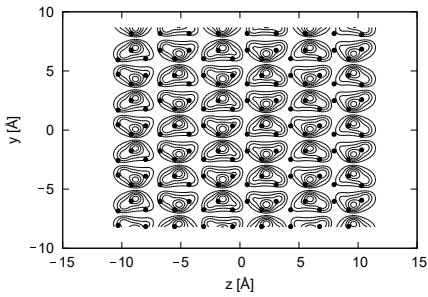

(d) $\theta=23^{\circ}$

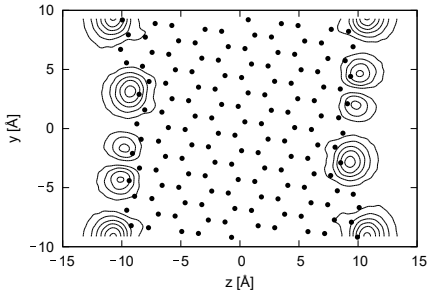

(b) $\theta=8^{\circ}$

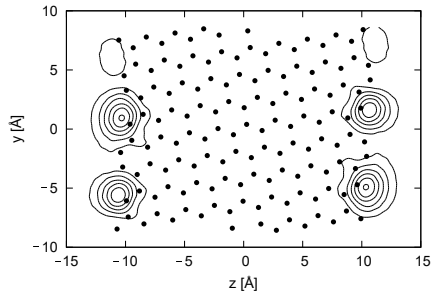

(e) $\theta=30^{\circ}$

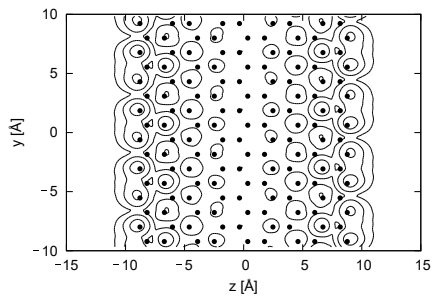

(c) $\theta=16^{\circ}$

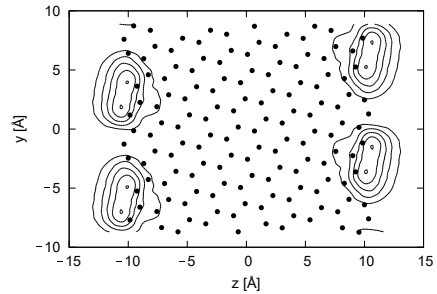

Figure 8: Contour plots of wave functions at the $\Gamma$ point near the $E_{F}$ of graphene nanoribbons with clean edges.

tra calculated by the local density approximation. Thus, the electronic structure modulation of ribbons with clean edges is also absent in the case of the local spin density approximation.

It is worth investigating the detailed properties of the flat band states that appeared around the $E_{F}$. To unravel the origin of the states, we calculate the wave function of the electron states near the $E_{F}$. Fig. 8 shows the squared wave function of nanoribbons with edge angles of $0,8,16,23$, and $30^{\circ}$ at the $\Gamma$ point near the $E_{F}$. In the case of the nanoribbon with armchair edges, the states extend throughout the ribbons, revealing their $\pi$ and $\pi^{*}$ natures. On the other hand, for nanoribbons with finite edge angles except $\theta=30^{\circ}$, the wave functions are localized and distributed aside on the edge atomic sites, especially constituting of zigzag shapes. Thus, the states are classified as dangling bond states arising from the unsaturated 
covalent bond of $\mathrm{C}$ atoms at the apex of the edge with zigzag shapes. It should be noted that such states are absent at the atomic sites belonging to the edge with armchair shapes. In this case, the dimerization of edge atoms leads to substantial upward and downward shift for states with antibonding and bonding natures, respectively. For nanoribbons with zigzag edges, we can see the hybridized nature of the wave function of edge states and that of dangling bond states because they are almost degenerate.

\subsection{Electrostatic potential and electric field}

It is worth investigating the detailed electrostatic potential and electric field in/around the graphene nanoribbons in terms of the edge shapes and edge termination. Figures 9 and 10 show the contour plots of the electrostatic potential of graphene nanoribbons with hydrogenated and clean edges, together with the corresponding electric field. For ribbons with hydrogenated edges, there is a substantial potential gradient inside the ribbon with armchair edges. As pointed above, the

potential gradient is ascribed to the bond alternation arising from the edge $\mathrm{C}-\mathrm{H}$ bonds. With increasing edge angle, the potential gradient inside the ribbon monotonically decreases. For instance, the sizable potential gradient is absent inside the ribbon with zigzag edges, and the potential drop only occurs at the edge $\mathrm{C}$ atoms.

The electric field outside the edges strongly depends on the edge shape and termination. In the case of hydrogenated edges, the electric field is strongly concentrated at the protuberant regions of edges where the armchair and zigzag edges coexist. Thus, field emission may occur at protuberances on the hydrogenated graphene nanoribbons $[50,51]$. By removing the hydrogen atoms, we found a different field distribution outside the edges: in contrast to the hydrogenated edges, the field concentrates at the atoms belonging to the zigzag edge close to the pro- 

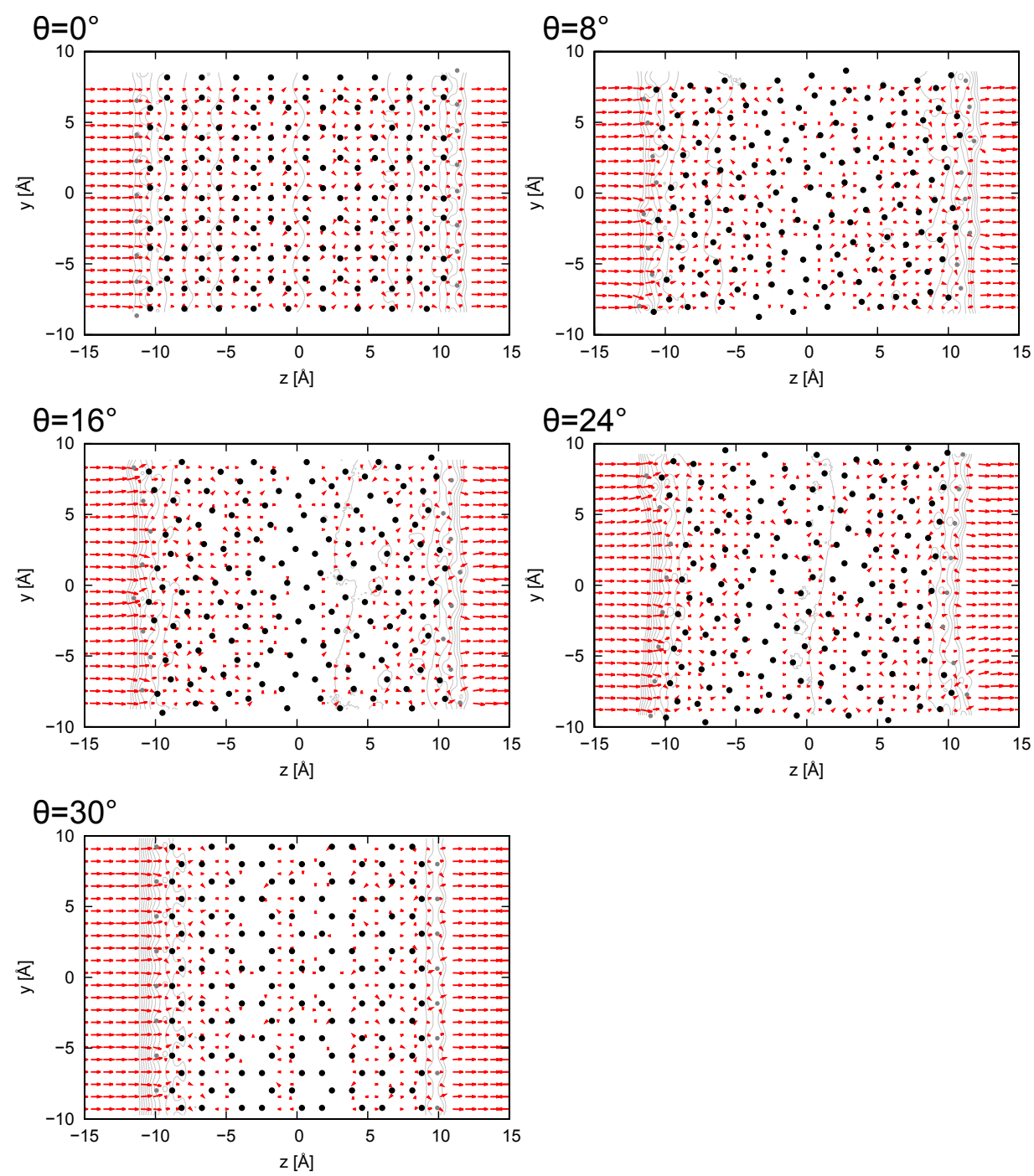

Figure 9: (Color online) Contour and vector plots of the electrostatic potential and electric field, respectively, of hydrogenated graphene nanoribbons with various edge angles under an external electric field. For both plots, the deep attractive potentials arising from nuclei are subtracted by taking the difference between the potentials with and without the external electric field. Black and gray circles denote $\mathrm{C}$ and $\mathrm{H}$ atoms, respectively. 

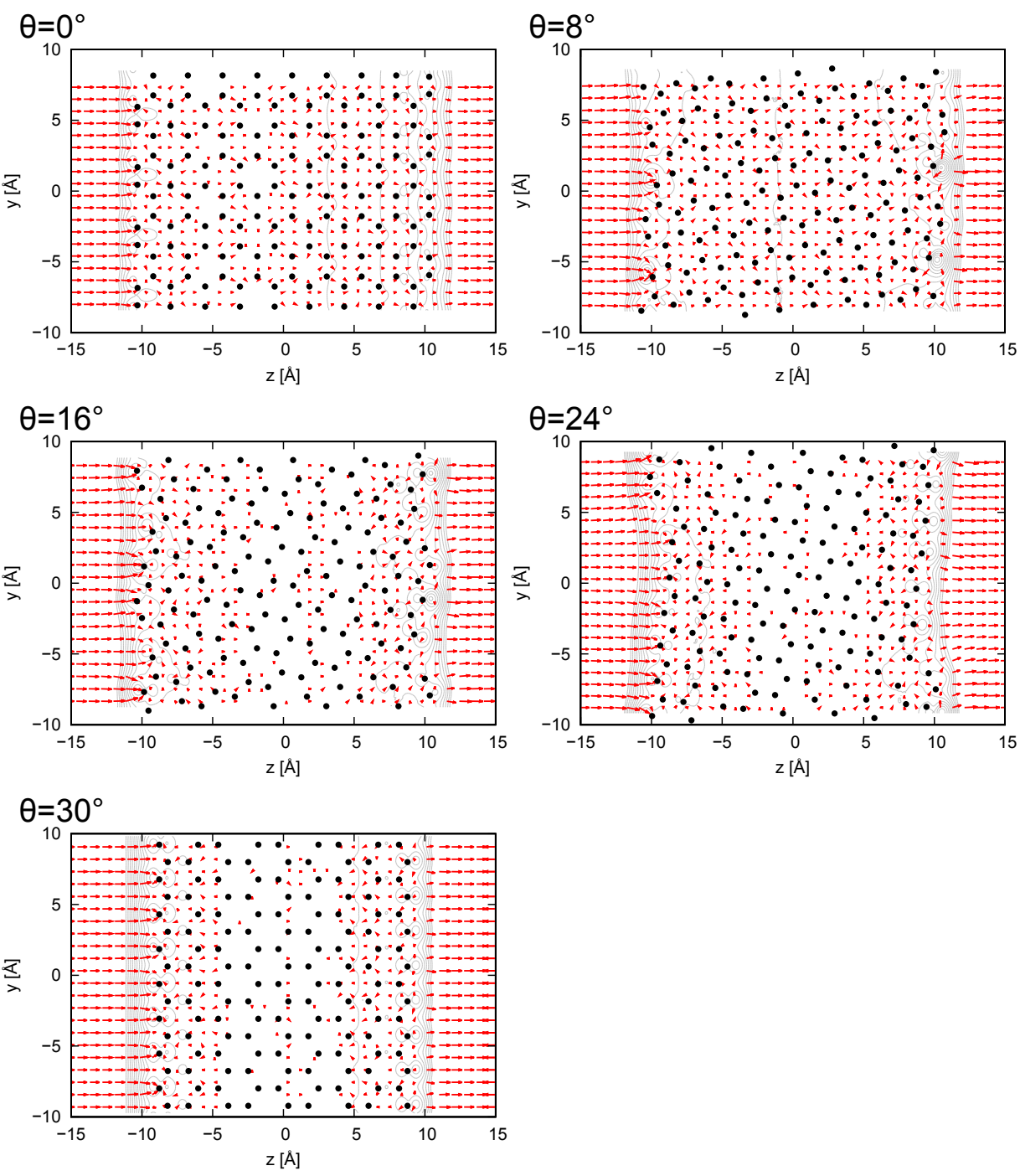

Figure 10: (Color online) Contour and vector plots of the electrostatic potential and electric field, respectively, of clean graphene nanoribbons with various edge angles under an external electric field. For both plots, the deep attractive potentials arising from nuclei are subtracted by taking the difference between the potentials with and without the external electric field. Black circles denote $\mathrm{C}$ atoms. 
tuberant region. In this case, edge reconstruction flattens the atomic sites at the convex region to reduce the energy cost arising from the dangling bonds. Therefore, the zigzag edges are dominant for field emission in graphene nanoribbons with clean edges.

\section{Summary}

We studied the geometric and electronic structures of graphene nanoribbons with edge angles from armchair to zigzag using density functional theory. Our calculations showed that the edge stability and electronic structure of graphene nanoribbons strongly depends on the detailed edge structures. The edge formation energy retains a constant value around the armchair edges, while it monotonically increases with increasing a portion of zigzag shapes. This fact indicates that graphene nanoribbons and nanoflakes inherently possess edge roughnesses within the angle deviation of $\pm 16^{\circ}$ around the armchair edges. Furthermore, the edge stability strongly correlates with the electronic structures of graphene nanoribbons: ribbons with near-armchair edges are semiconductors with a finite energy gap, while ribbons with near-zigzag edges are metals in which the density of states at the $E_{F}$ monotonically increases with increasing a portion of zigzag edges. By analyzing the wave functions near the $E_{F}$, we found that the edge state soon appeared around the $E_{F}$, even though the nanoribbons do not have perfect zigzag edges. Based on these facts, we conclude that edge states induced by the zigzag portion of the graphene edges crucially determine the stability of the edge structure of nanoribbons and nanoflakes of graphene. We further demonstrated that a parallel electric field decreases the edge formation energy of all graphene nanoribbons studied here. For ribbons with clean edges, because the dangling bond states 
appeared near the $E_{F}$, the edge formation energy monotonically increases with increasing a portion of zigzag edges.

We also investigate the electric field profile around the edge atomic sites. In the case of edge termination by hydrogen atoms, the electric field is concentrated at the protuberant region corresponding with the boundary of zigzag and armchair edges. In sharp contrast, the field is concentrated at the edge atomic sites with zigzag shapes for clean edges. These facts give theoretical insights in the practical application of graphene edges for field emission devices.

\section{5. acknowledgments}

One of the authors, AY, acknowledges a Grant-in-Aid for JSPS Fellows. This work was partially supported by CREST, the Japan Science and Technology Agency, and a Grant-in-Aid for Scientific Research from the Ministry of Education, Culture, Sports, Science and Technology of Japan.

[1] Dresselhaus MS, Dresselhaus G. Intercalation compounds of graphite. Adv. Phys. 1981;30(2):139-326.

[2] Neto AHC, Guinea F, Peres NMR, Novoselov KS, Geim AK. The electronic properties of graphene. Rev. Mod. Phys. 2009;81(1):109-62.

[3] Seyller Th, Emtsev KV, Gao K, Speck F, Ley L, Tadich A, Broekman L, Riley JD, Leckey RCG, Rader O, Varykhalov A, Shikin AM. Structural and electronic properties of graphite layers grown on $\mathrm{SiC}(0001)$. Surf. Sci. 2006;600(18):3906-11. 
[4] Novoselov KS, Geim AK, Morozov SV, Jiang D, Katsnelson MI, Grigorieva IV, Dubonos SV, Firsov AA. Two-dimensional gas of massless Dirac fermions in graphene. Nature 2005;438:197-200.

[5] Zhang Y, Tan Y-W, Stormer HL, Kim P. Experimental observation of the quantum Hall effect and Berry's phase in graphene. Nature 2005;438:201-4.

[6] Du X, Skachko I, Duerr F, Luican A, Andrei EY. Fractional quantum Hall effect and insulating phase of Dirac electrons in graphene. Nature 2009;462:192-5.

[7] Bolotin KI, Ghahari F, Shulman MD, Stormer HL, Kim P. Observation of the fractional quantum Hall effect in graphene. Nature 2009;462:196-9.

[8] Okada S. Semiconducting Electronic Structure of Graphene Adsorbed on Insulating Substrate: Fragility of the Graphene Linear Dispersion Band. Jpn. J. Appl. Phys. 2010;49(2R):020204.

[9] Cuong NT, Otani M, Okada S. Semiconducting Electronic Property of Graphene Adsorbed on (0001) Surfaces of $\mathrm{SiO}_{2}$. Phys. Rev. Lett. 2011;106:106801.

[10] Kamiya K, Umezawa N, Okada S. Energetics and electronic structure of graphene adsorbed on $\mathrm{HfO}_{2}(111)$ : Density functional theory calculations. Phys. Rev. B 2011;83:153413.

[11] Takagi Y, Okada S. Electronic Structure Modulation of Graphene by Metal Electrodes. Jpn. J. Appl. Phys. 2012;51(8R):085102. 
[12] Takagi Y, Okada S. Modulation of Electron-States of Graphite Thin Films by the Nearly Free Electron States of Metal Surfaces. Jpn. J. Appl. Phys. 2012;51(10R):100203.

[13] Koshino M, Ando T. Orbital diamagnetism in multilayer graphenes: Systematic study with the effective mass approximation. Phys. Rev. B 2007;76:085425.

[14] Koshino M, Ando T. Magneto-optical properties of multilayer graphene. Phys. Rev. B 2008;77:115313.

[15] Otani M, Koshino M, Takagi Y, Okada S. Intrinsic magnetic moment on (0001) surfaces of rhombohedral graphite. Phys. Rev. B 2010;81:161403(R).

[16] Otani M, Koshino M, Takagi Y, Okada S. Phase control of magnetic state of graphite thin films by electric field. Appl. Phys. Lett. 2010;96:242504.

[17] McCann E. Asymmetry gap in the electronic band structure of bilayer graphene. Phys. Rev. B 2006;74:161403.

[18] Castro EV, Novoselov KS, Morozov SV, Peres NMR, Lopes dos Santos JMB, Nilsson J, Guinea F, Geim AK, Neto AHC. Biased Bilayer Graphene: Semiconductor with a Gap Tunable by the Electric Field Effect. Phys. Rev. Lett. 2007;99:216802.

[19] Oostinga JB, Heersche HB, Liu L, Morpurgo AF, Vandersypen LMK. Gate-induced insulating state in bilayer graphene devices. Nature Mater. 2008;7:151-7. 
[20] Miyazaki H, Tsukagoshi K, Kanda A, Otani M, Okada S. Influence of Disorder on Conductance in Bilayer Graphene under Perpendicular Electric Field. Nano Lett. 2010;10(10):3888-92.

[21] Otani M, Okada S. Field-Induced Free-Electron Carriers in Graphite. J. Phys. Soc. Jpn. 2010;79(7):073701.

[22] Konabe S, Okada S. Robustness and Fragility of a Linear Dispersion Band of Bilayer Graphene under an Electric Field. J. Phys. Soc. Jpn. 2012;81(11):113702.

[23] Fujita M, Wakabayashi K, Nakada K, Kusakabe K. Peculiar Localized State at Zigzag Graphite Edge. J. Phys. Soc. Jpn. 1996;65(7):1920.

[24] Nakada K, Fujita M, Dresselhaus G, Dresselhaus MS. Edge state in graphene ribbons: Nanometer size effect and edge shape dependence. Phys. Rev. B 1996;54(24):17954-61.

[25] Miyamoto Y, Nakada K, Fujita M. First-principles study of edge states of H-terminated graphitic ribbons. Phys. Rev. B 1999;59(15):9858-61.

[26] Kobayashi Y, Fukui K, Enoki T. Edge state on hydrogen-terminated graphite edges investigated by scanning tunneling microscopy. Phys. Rev. B 2006;73:125415.

[27] Hashimoto A, Suenaga K, Gloter A, Urita K, Iijima S. Direct evidence for atomic defects in graphene layers. Nature 2004;430:870.

[28] Ma Y, Lehtinen PO, Foster AS, Nieminen RM. Magnetic properties of 
vacancies in graphene and single-walled carbon nanotubes. New J. Phys. 2004;6:68.

[29] Okada S, Oshiyama A. Magnetic Ordering in Hexagonally Bonded Sheets with First-Row Elements. Phys. Rev. Lett. 2001;87(14):146803.

[30] Kusakabe K, Maruyama M. Magnetic nanographite. Phys. Rev. B 2003;67:092406.

[31] Shimomura Y, Takane Y, Watanabe K. Electronic States and Local Density of States in Graphene with a Corner Edge Structure. J. Phys. Soc. Jpn. 2011;80:054710.

[32] Cuong NT, Otani M, Okada S. Absence of edge states near the $120^{\circ}$ corners of zigzag graphene nanoribbons. Phys. Rev. B 2013;87:045424.

[33] Barone V, Hod O, Scuseria GE. Electronic Structure and Stability of Semiconducting Graphene Nanoribbons. Nano Lett. 2006;6(12):2748-54.

[34] Okada S. Energetics of nanoscale graphene ribbons: Edge geometries and electronic structures. Phys. Rev. B 2008;77:041408.

[35] Huang B, Liu M, Su N, Wu J, Duan W, Gu BL, Liu F. Quantum Manifestations of Graphene Edge Stress and Edge Instability: A First-Principles Study. Phys. Rev. Lett. 2009;102:166404.

[36] Koskinen P, Malola S, Häkkinen H. Self-Passivating Edge Reconstructions of Graphene. Phys. Rev. Lett. 2008;101:115502.

[37] Gan CK, Srolovitz DJ. First-principles study of graphene edge properties and flake shapes. Phys. Rev. B 2010;81:125445. 
[38] Suda S, Oshiyama A. Energetics, Electron States, and Magnetization in Nearly Zigzag-Edged Graphene Nano-Ribbons. J. Phys. Soc. Jpn. 2015;84(2):024704.

[39] Hohenberg P, Kohn W. Inhomogeneous Electron Gas. Phys. Rev. 1964;136(3B):B864-71.

[40] Kohn W, Sham LJ. Self-Consistent Equations Including Exchange and Correlation Effects. Phys. Rev. 1965;140(4A):A1133-8.

[41] Morikawa Y, Iwata K, Terakura K. Theoretical study of hydrogenation process of formate on clean and $\mathrm{Zn}$ deposited $\mathrm{Cu}(111)$ surfaces. Appl. Surf. Sci. 2001;169-170:11-15.

[42] Perdew JP, Zunger A. Self-interaction correction to density-functional approximations for many-electron systems. Phys. Rev. B 1981;23(10):504879.

[43] Ceperley DM, Alder BJ. Ground State of the Electron Gas by a Stochastic Method. Phys. Rev. Lett. 1980;45(7):566-9.

[44] Vanderbilt D. Soft self-consistent pseudopotentials in a generalized eigenvalue formalism. Phys. Rev. B 1990;41(11):7892-5.

[45] Otani M, Sugino O. First-principles calculations of charged surfaces and interfaces: A plane-wave nonrepeated slab approach. Phys. Rev. B 2006;73:115407.

[46] Son Y-W, Cohen ML, Louie SG. Half-metallic graphene nanoribbons. Nature 2006;444:347-9. 
[47] Dutta S, Manna AK, Pati SK. Intrinsic Half-Metallicity in Modified Graphene Nanoribbons. Phys. Rev. Lett. 2009;102:096601.

[48] Yamanaka A, Okada S. Structural dependence of electronic properties of graphene nanoribbons on an electric field. Jpn. J. Appl. Phys. 2014;53:06JD05.

[49] Kawai T, Miyamoto Y, Sugino O, Koga Y. Graphitic ribbons without hydrogen-termination: Electronic structures and stabilities. Phys. Rev. B 2000;62:R16349.

[50] Tada K, Watanabe K. Ab Initio Study of Field Emission from Graphitic Ribbons. Phys. Rev. Lett. 2002;88:127601.

[51] Araidai M, Nakamura Y, Watanabe K. Field emission mechanisms of graphitic nanostructures. Phys. Rev. B 2004;70:245410. 